\title{
Assessing the impact of nursing training on patient safety in neonatal intensive care unit at maternity and children's hospital in the K.S.A.
}

\author{
Nashi Masnad Alreshidi*1, Michelle Garcia ${ }^{2}$, Rana Mohammad Al Aseeri ${ }^{3}$, Talal Majed Al Reshedi ${ }^{4}$ \\ ${ }^{1}$ Continuing Nursing Education, Regional Nursing Administration, Hail, Saudi Arabia \\ ${ }^{2}$ Regional Nursing Quality Mangement, Hail, Saudi Arabia \\ ${ }^{3}$ Regional Nursing Administration, General Directorate of Health Affairs, Hail, Saudi Arabia \\ ${ }^{4}$ Research and Planning Department, General Directorate of Health Affairs, Hail, Saudi Arabia
}

Received: March 8, 2020

DOI: $10.5430 /$ jnep.v10n7p53
Accepted: April 6, 2020

Online Published: April 14, 2020

\begin{abstract}
Background and objective: Nursing training programs with thorough planning and implementation, staff knowledge, skills and attitude is enhanced thereby contributing to an improved performance, teamwork and commitment towards achieving organizational goals and objectives. This is a descriptive report of a previously conducted intervention at the hospital. This study seeks to understand the level of staff perception, on the effectiveness of the training/courses implemented or conducted and its impact on staff performance, morale and attitude.

Methods: The sample of this study was collected by cluster sampling method which includes 88 NICU staff nurse in Maternity and Children's Hospital of 38 bed capacity. This is a descriptive report of a previously conducted intervention at the hospital. A 5-point likert scale was self-report evaluation tool to assess training effectiveness of the implemented courses and or training and mean score is computed and interpreted. Contents of the questionnaire from the federal authority for government human resources is subjected for content validation.

Results: A total of 88 NICU staff nurses, ages ranged between 26-31, majority hold the bachelor degree 77 (87.5\%), with an average clinical experience between 1-4 years. According to the 15 enlisted courses/trainings implemented in NICU, the highest rate of attendance is Neonatal Resuscitation Program 60 (68.2\%) next is S.T.A.B.L.E. Program 74 (84.1\%) and third N.I.D.C.A.P. $48(54.5 \%)$. And base on the self-reported evaluation on the level of agreement of the 6 item question scored by 5-point likert scale the result has a mean score of $4=$ agree. Majority of the respondents, $71(80.9 \%)$ had the chance to make use of the skills learnt while 17 (19\%) had no chance due to feeling busy most of the time $8(10 \%)$.

Conclusions and implication: It is concluded that to ensure patient safety, proper education and correct implementation of training and evaluation results into a positive outcome. A positive outcome on job performance, staff morale and teamwork toward the achievement of organizational goals and objectives.
\end{abstract}

Key Words: Impact, Training, Effectiveness, Patient safety, Neonatal intensive care unit, Nurse

\section{INTRODUCTION}

Patient safety and quality is a global issue in healthcare industry. It is the priority in preventing medical error before it causes death, harm and injury to a patient. Considering patient in critical care unit, specializing in the supervision of neonates (first 28 days of life) where complex medical

\footnotetext{
*Correspondence: Nashi Masnad Alreshidi; Email: nmalreshidi@moh.gov.sa; Address: Continuing Nursing Education, Regional Nursing Administration, Hail, Saudi Arabia.
} 
care ${ }^{[1]}$ management and environment pose the increase risk of medical errors thereby special training and intensive educational program are equally important. Maintaining an educational and training qualification of nurses working in specialized area is significantly required. In line with the fast trend and emerging technology in nursing practice, nurses must be inclined and technologically adept to ensure effective, safe and quality delivery of nursing care.

Assessment of nursing training on patient safety is one of the foundations to improve education and training programs conducted to a specific unit in the hospital. Therefore, an evaluation and evaluation methods must be conducted to find an area that needs improvement and to maintain a culture of safe and effective delivery of patient care.

In the United States, employee training and education allocation is increasing by $14 \%$ every year because it has been proven that enhancing knowledge, skills and measuring training effectiveness are equally relevant to improve staff engagement and retention. Performance indicators of past training also act as critical parameters when planning future workshops. ${ }^{[2]}$

\section{BACKGROUND}

\subsection{Patient safety and nursing care}

Among the 12 dimensions used to measure patient safety culture weakness were as follows: management support for patient safety, teamwork, occurrence variance reports, open communication, supervisor/manager expectations, actions promoting patient safety hospital handoffs and transitions and non-punitive response to errors and overall perception of patient safety. ${ }^{[3]}$ It is evident that the higher the RN staffing ratio the lower the patient mortality rate. ${ }^{[4]}$ and it is known that $70 \%$ of mistakes in health care are due to human errors. [5]

\subsection{Nursing education and patient safety}

Nurses make-up the largest portion of healthcare professionals and in proper collaboration with patient and their families checking procedures, learning from errors and effective communication with other members of the healthcare team all contributes to improve patient safety including education and training of healthcare professionals are significantly needed. ${ }^{[6]}$

In the study of "Using simulation-based training to improve patient: what does it take." It was concluded that the healthcare community can gain significantly from using simulation based-training to reduce errors and improve patient safety when it is designed and delivered appropriately. ${ }^{[7]}$

However, in the study of "Academic training of nursing pro- fessionals and its relevance to the workplace" $53.8 \%$ perceived training as irrelevant to the needs of the workplace and $94.2 \%$ reported that academic education impacts the quality of care provided. ${ }^{[8]}$

Consequently, the TeamSTEPPS simulation-based curriculum: from needs assessment to delivery and evaluation. It was determined when needs assessment has been conducted and evaluated it can lead to more learning thus leading to change in practice. It was described that "simulation based training is generally very positive, and the simulations are regarded as realistic and perceived as useful in helping the learner to reflect". A significant improvement on theoretical and clinical skills of participants in neonatal resuscitation were described when the training focuses on the following resuscitation guidelines and practical skills, decision making and team skills were all given emphasis ${ }^{[9]}$ to build a foundation of knowledge and best practice. ${ }^{[10]}$

In terms of patients' outcome, sufficient number of nurses in acute care areas can reduce the risk of patient mortality in high income countries and that it is non-definitive in nurse: patient ratio while there is a limited evidence that expert nurses can produce health outcomes and low to average information for parental support programs conveyed by nurses including home visit on improving function and other health service outcomes for older people all impacts a good patient outcome. $^{[11]}$

In identifying training needs during the training process with clear training objectives, content and application of job training techniques could significantly influence job performance and employees are likely to perform in their respective tasks on job. ${ }^{[12]}$

The Kirkpatrick Evaluation Model had four level of measurement: ${ }^{[2]}$ Level 1 is Reaction which assesses learner's reaction to the training, its importance and its practicality and where feedback on the learning experience is gathered before and after the training. Level 2 - Learning; it is the level to gauge knowledge and skills obtained by learners, Level 3- Behavior to recognize the impact of training on learner's performance and attitude at work. And Level 4 - Results; in which tangible results such as cost, quality improvement, project completion, productivity employee retention and etc. are evaluated.

Furthermore, training has been proven to develop performance improvement for the employee and organization by positively influencing employee performance by developing employee knowledge, skills, ability, competencies and behavior. ${ }^{[13]}$ Indeed, it also facilitates organization to determine non-performing employees and thus, knowledge, skills 
and attitudes needs to be molded, thus promoting employee competence enabling them to perform their job efficiently, and achieve objectives in a competitive manner. ${ }^{[14]}$

\subsection{Patient safety in Saudi Arabia}

Patient safety culture in Saudi Arabia was graded excellent or very good by $60 \%$ of respondents, acceptable by $33 \%$ and failing or poor by $7 \%, 63 \%$, claims that patient safety is always observed and that $70 \%$ affirms the standard procedures that prevents errors from happening. On the other hand, about one third of the respondents perceived that very less serious mistakes happens in their hospitals. ${ }^{[15]}$

Moreover, in Saudi Arabia areas of strength were identified such as: unit teamwork, continuous learning improvement, management support for patient safety and communication about error. Whereas, the major area of strength identified were the process of the hospital to improve patient safety and teamwork within the unit. Respondents claimed that all staff support each other within the unit, and work together as a team. As highlighted within the composite on Hospital Management Support for Patient Safety, $86.9 \%$ of respondents indicated that hospital management prioritizes patient. ${ }^{[16]}$

\section{METHODS AND PROCEDURES OF THE STUDY}

\subsection{Study design}

This descriptive quantitative study assessed the impact of nursing training on patient safety in neonatal intensive care unit. Nurses enrolled in these training program in the past were subject to participate in the study. Nursing training consist of theoretical and practical training provided to staff with the purpose to enhance staff knowledge, skills and attitude to be more efficient in the performance of their duties and responsibilities.

\subsection{Study settings}

The study was conducted in neonatal intensive care unit in Maternity and Children's Hospital of 38 bed capacity. It is the largest referral hospital for neonates that need intensive care.

\subsection{Study population and sample}

The Cluster Sampling Method was used to recruit the sample. The inclusion criteria were all nurses working in NICU, regardless of age, nationality, qualification and years of experience in NICU. All staff had given a chance to answer the questionnaire. Staff nurses on duty had given an ample time to answer the questionnaire, while nurses not on duty answered the questionnaire at their most convenient time. Other nurses working in other departments were excluded in the sample.

\subsection{Measurement tools}

The Researchers used a survey questionnaire adopted from the federal authority for government human resources in gathering the data needed to assess the effectiveness of training/courses provided in NICU. The first part used to determine the training/courses implemented and attended by NICU staff. Courses implemented based on the needs of the unit were enlisted and attendance of staff nurses in the unit were calculated by percentage. While the second part, is the self-report evaluation of the staff determining the effectiveness of the training/course. It uses a 5-point likert scale composed of 6 questions: Improvement on job performance, chance to make use of the skills they learnt, and application of information into practice, confidence, willingness and ability to mentor others. These questions were scored from 5 as strongly agree 4 agree, 3 neither agree nor disagree, 2 disagree and 1 strongly disagree. The respondents chance to apply in NICU the skills they've learnt were also evaluated by an answer YES or NO. And follow-up question was given if their answer is NO, to determine the reasons of not having the chance.

In the present study, reliability of the questionnaire is subjected for content validation and this process was performed by a panel of experts in nursing research in which item was assesses for its usefulness and its relevancy to the selected objectives.

\subsection{Study procedures}

Prior to commencement of the study, an approval was obtained from the IRB and to all involved organizations. The questionnaire was sent electronically to Director of Nursing and communicated to Nursing Quality Coordinator for follow up to the Head of NICU and staff nurses.

Inclusion criteria (all NICU staff nurses and Head Nurse) and exclusion criteria (staff nurses working at different units) of the study were discussed to the Director of Nursing and its purpose. At a different time setting, respondents participated voluntarily in the study at their most convenient time. The information provided by the respondents was completely treated anonymously and no names or identifying numbers were collected on any of the instruments.

\subsection{Data analysis}

The Statistical Package for Social Sciences version 19 was used for data analysis. All variables were coded and checked for the normality of the distributions and for the measurement scale's reliability, Cronbach's $\alpha$ was calculated.

Descriptive statistics were performed for an overview of the 
demographic and educational data. In addition, continuous data are presented as mean, range and standard deviations and categorical data are presented as percentages and numbers.

\subsection{Ethics approval and consent to participate}

This research was approved by the Ethics Committee of Hail Region with IRB Log Number 2020-6. Verbal consent was obtained to the respondents before data collection. Questionnaires were completed anonymously to ensure confidentiality.

\section{Results}

\subsection{Demographic characteristics}

Demographic characteristics is presented in Table 1. Exactly 88 NICU staff nurses participated in the study, ages ranged between $26-31$ years, about $87.5 \%$ hold the bachelor degree while $12.5 \%$ hold the diploma degree and in terms of work experience in NICU, more than $71 \%$ were working in the NICU between $1-4$ years, $12.5 \%$ above 10 years, while $10.2 \%$ had $5-9$ years of experience and more than $5 \%$ belongs to 6 months above.

\subsection{Training/Courses attended}

Training/courses required and conducted in NICU based on the pre-training needs assessment of the unit. Table 2 shows the implemented training/courses in neonatal inten- sive care unit. The percentages show that the majority of nurses underwent S.T.A.B.L.E. Program $84.1 \%$, care of the neonates on ventilator $75 \%$ and assisting in endotracheal intubation $70.5 \%$. Consequently, there were $68.2 \%$ attended NRP, $54.5 \%$ in NIDCAP, and blood exchange transfusion $64.8 \%$. Meanwhile, nurses attended the Adherence to standards in medication administration $45.5 \%$, Recognizing critical ECG changes including arrthymias in the neonates $35.2 \%$ and Implementing risk management in Nursing 33\%. Moreover, there were $31.8 \%$ attended The Influence of EvidencedBased Practices in the Nursing Evolution and 28.4\% in Communicable and emerging infectious diseases. As can be seen, the lowest three went to the impact of maternal nutrition in the fetus $27.3 \%$, Nurse Proficiency in managing obstetric emergencies $26.1 \%$ and Management of patients with reproductive disorders $25 \%$.

Table 1. Characteristics of the respondents and descriptive results

\begin{tabular}{|c|c|c|c|}
\hline \multicolumn{4}{|c|}{ Demographic Characteristics $N=88$} \\
\hline \multicolumn{2}{|c|}{ Age, mean (range) } & \multicolumn{2}{|c|}{$40(26-31)$} \\
\hline & & $t$ & $\%$ \\
\hline \multirow{2}{*}{ Qualifications, \% } & Diploma & 11 & 12.5 \\
\hline & Bachelor & 77 & 87.5 \\
\hline \multirow{4}{*}{$\begin{array}{l}\text { Years of } \\
\text { experience in } \\
\text { NICU, \% }\end{array}$} & 6 months above & 5 & 5.7 \\
\hline & $1-4$ years & 63 & 71.6 \\
\hline & 5-9 years & 9 & 10.2 \\
\hline & 10 years above & 11 & 12.5 \\
\hline
\end{tabular}

Table 2. Trainings courses attended

\begin{tabular}{lll}
\hline PROGRAM & $\mathbf{\%}$ & 68.2 \\
\hline Neonatal Resuscitation Program (NRP) & 60 & 84.1 \\
S.T.A.B.L.E. Program & 74 & 54.5 \\
The Newborn Individualized Developmental Care and Assessment Program (NIDCAP) & 48 & 27.3 \\
The Impact of Maternal Nutrition in the Fetus & 24 & 33 \\
Implementing Risk Management in Nursing & 29 & 45.5 \\
Adherence to Standards in Medication Administration & 40 & 25 \\
Management of Patients with Reproductive Disorders & 22 & 26.1 \\
Nurse Proficiency in Managing Obstetric Emergencies & 23 & 31.8 \\
The Influence of Evidenced-Based Practices in the Nursing Evolution & 28 & 28.4 \\
Communicable and Emerging Infectious Diseases & 25 & 64.8 \\
Blood Exchange Transfusion & 57 & 67 \\
Assisting Survanta Administration \&Equipment used & 59 & 75 \\
Care of the neonates on Ventilator & 66 & 70.5 \\
Assisting in Endotracheal Intubation & 62 & 35.2 \\
Recognizing Critical ECG changes including arrthymias in the neonates & 31 & Pediatric Advance Life Support (PALS), \\
Other Courses Attended by the respondents & Conscious sedation, Care of pre-term \\
& babies, Management of Neonatal Jaundice \\
\hline
\end{tabular}




\subsection{Effectiveness of training/courses implemented}

The distribution of participant's responses base on the selfreport evaluation on the effectiveness of training or courses in NICU as shown in Figure 1, the frequency show most of the respondents agreed on the benefits provisions of the NICU training which was evidenced by selecting "agree" and "strongly agree" by the majority of nurses.

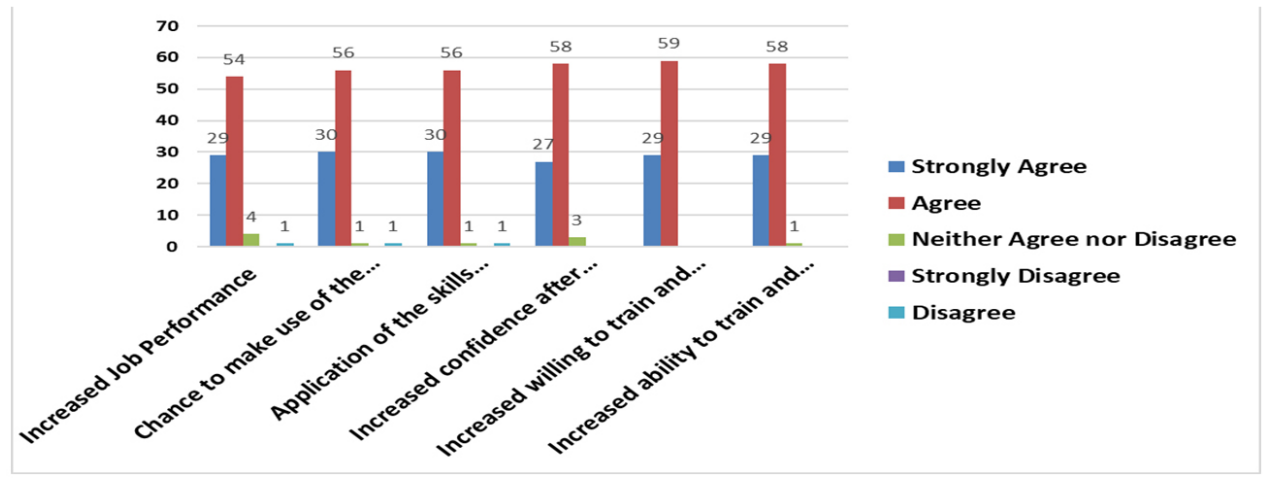

Figure 1. Frequency of staff Level of agreement on training effectiveness

4.4 Chance of the respondents to make use of the skills and reasons for not having a chance to make use of the skills learnt

Table 3 present the distribution of chance of the respondents to make use of the knowledge and skills acquired. It signifies that $80.9 \%$ of the respondents had a positive reaction and behavior towards the program or courses and training while about $19 \%$ had no chance due to feeling busy most of the time.

Table 3. Respondents chance to make use of the skills acquired

\begin{tabular}{ll}
\hline YES (\%) & NO (\%) \\
\hline (72) $80.9 \%$ & $16(19.1 \%)$ \\
\hline REASONS & \\
\hline I was very busy & $10(11 \%)$ \\
I have not had the chance & $5(7 \%)$ \\
I have not learned anything & $1(1 \%)$ \\
\hline
\end{tabular}

\subsection{State of training effectiveness}

The weighted mean of each dimensions are reported in Table 4. Six (6) criteria were use assess the impact of nursing in a scale of 5 (strongly agree) 1 (disagree). The total mean score in all dimension was 4 was interpreted as agree.

\section{Discussion}

Southeast Asian countries had inter-related quality on patient safety concerns impacting the delivery of care. It was evident as high incidence rate of medication errors subdivided into different categories such as: administration error (59\%), transcription (15\%), dispensing (14\%) and prescribing error. ${ }^{[17]}$ In this study, part of the nursing training, "Adherence to Standards in Medication Administration was conducted where, $45.5 \%$ of the sample attended the training.

Table 4. Training effectiveness

\begin{tabular}{lll}
\hline Criteria & Mean & Interpretative \\
\hline $\begin{array}{l}\text { My job performance level has raised as a } \\
\text { result of my attendance in the training } \\
\text { course. }\end{array}$ & 4 & Agree \\
$\begin{array}{l}\text { I had the chance to make the best use of } \\
\text { the skills I learnt in the training course. }\end{array}$ & 4 & Agree \\
$\begin{array}{l}\text { I can apply the information in my } \\
\text { practice/service setting }\end{array}$ & 4 & Agree \\
$\begin{array}{l}\text { As a result of this training, I feel more } \\
\text { confident to perform my task. }\end{array}$ & 4 & Agree \\
$\begin{array}{l}\text { Increasing my willingness to train and } \\
\text { mentor others }\end{array}$ & 4 & Agree \\
$\begin{array}{l}\text { Increasing my ability to train and mentor } \\
\text { others. }\end{array}$ & 4 & Agree \\
Mean & 4 & Agree \\
\hline
\end{tabular}

Last 2012 in Cambodia study, the most common problem in healthcare is maternal and peri and post-natal care as well as the lack of skills in neonatal resuscitation are apparent. ${ }^{[17]}$ Interventions included in this study relevant to these findings were: N.R.P, S.T.A.B.L.E, Program, The NIDCAP, The Impact of Maternal Nutrition in the Fetus, Nurse Proficiency in Managing Obstetric Emergencies.

Nursing training on Communicable and Emerging Infectious Diseases attended by $28.4 \%$ (25) of the NICU nurses was conducted. Whereas in Indonesia, lack of awareness on HAI was evident. There were no clear guidelines to disseminate information regarding this issue and evidence-based knowledge not used due to restricted resources and cultural or societal norms. ${ }^{[17]}$

Whereas, in Philippines, last 2011 there were 199 identified 
causes of HAIs namely, ventilator devices, central line and urinary catheter. ${ }^{[17]}$ In line with these findings, related procedures and equipments used in NICU had given a priority in building the training program. Education and training on Blood Exchange Transfusion, Care of the neonates on Ventilator and Assisting in Endotracheal Intubation.

When respondents ask about their perception on the factors affecting patient safety management practices in middle east, responses were: heavy workload issues $87 \%^{[16]}$ that was similar to this study where $10 \%$ of nurses felt they were too busy to apply the skills they learnt in nursing training program. It was found out that through conferences, seminars and workshops enhances nurse's self-confidence, independence job performance. ${ }^{[18]}$ That clearly supports the result of this study where, $30.6 \%$ (27) strongly agree and $65.9 \%$ (58) agree with a mean score of 4 interpretative as AGREE on the item "I feel more confident to perform my task." The findings of this study when measured the reaction of respondents which is the Level 1 when assessing the effectiveness of training base on Kirkpatrick Evaluation Model for evaluating training, indicates that the training/courses administered for NICU had a positive impact in terms of the relevance and usefulness of the training as evidenced by a mean of 56.83 at a level of "agree" in the 5-point likert scale.

At Level 2-learning criteria, 54 out of 88 respondents agreed that their level of job performance significantly increase as a result of the attendance at the training and courses and these result is consistent when compared to the previous of study in South Korea where it showed that participants who had scored significantly higher on overall PS competencies, skills, and knowledge after undergoing patient safety education.

Moreover, evaluating at Level 3-behavior criteria which measures the effectiveness and impact of training on job performance, it is clearly illustrated that 58 out of 88 were willing to train and mentor others that is supported by the data of $66 \%$ claims that their level of confidence was enhanced after attending the training and courses. This results were strengthened in the study of Truit D, where it was found out that there is a direct relationship between training experience, experience and proficiency. ${ }^{[19]}$

Finally, the study reveals that respondents strongly agreed on the criteria mentioned to evaluate the effectiveness of the courses and trainings listed in Table 2. As mentioned, medical errors impact 1 in 10 patients worldwide; ${ }^{[20]}$ and consequently medication errors are due to wrong patient, wrong or delayed diagnosis and administration or method used for treatment and most common types of medication errors are those of dosing, prescribing, frequency and route of administration. Furthermore, it was also cited that wrong drug infusion rate via pumps, packaging/labelling of drugs, excess oxygen therapy, human factors, miscommunication and equipment/delivery devices failures, patient misidentification and false documentation were all identified as causes of medication errors. ${ }^{[21]}$ These topics were clearly illustrated in Table 2 as implemented courses for NICU staff; as reflected on their self-reported evaluation on a mean score of 4 interpreted as agree; nurses deemed it necessary and considered it as effective.

All findings are reflective on staff knowledge, skills and attitude with the goal to raise awareness on the importance of patient safety through the educational programs implemented in the unit.

\section{Limitations}

This study has the following limitations. The measured level of assessing the effectiveness of training and courses may somewhat vary from the actual level, since it was assessed with a self-report scale and the reporting instrument may have decreased the reliability of the responses due to misinterpretation of some of the items. Nevertheless, these limitations, the findings of the study provide important contribution to the existing research particularly for neonatal intensive care unit nurses.

\section{CONCLUSION AND RECOMMENDATIONS}

This study offers an understanding that when nurses had given a proper education, training and chance to make use of the skills achieved as a result of training or courses attended, chances are these staff nurses would have a positive attitude towards mentoring and training other nurses. This study found that training courses are required and should be administered to ensure safe and effective care were significantly needed by the respondents in which was supported by the data showing $71.6 \%$ had 1-4 years' experience which is considered advanced beginner where knowledge is still developing. With these educational programs, it increases their level of confidence to render care, equally enhanced knowledge and skills and resulting a boost of morale for nurses.

The significance of this study are as follows: First, it provides the over-all picture of training interventions in the unit to ensure patient safety. Second, it reveals the impact on advanced beginner nurses as highly effective in terms of professional growth and development. Lastly, the findings imply the need for adequate supportive environment, manpower and effective communication.

\section{ACKNOWLEDGEMENTS}

This research would like to thank all nurses who partici- 
pated the survey, the Director of Nursing, Nursing Quality Supervisor, Head Nurse of NICU who assisted to make this study possible. This study would have had not been possible without their participation and assistance.

\section{CONFLICTS OF INTEREST DISCLOSURE}

The authors expressed they have no personal interest on the outcome of the research and will not use at their own advantage.

\section{REFERENCES}

[1] Raju TN, Suresh G, Higgins RD. Patient Safety in the Context of Intensive Care: Research and Educational Opportunities. Pediatric Research. 2011; 70(1): 109-15. PMid:21386749 https: //doi.org/10.1203/PDR.0b013e3182182853

[2] Verma E. How to measure the effectiveness of corporate training. December 18, 2019.

[3] Alenezi A, Pandaan RP, Almazan J. Clinical practitioners' perception of the dimensions of patient safety culture in a government hospital: A one-sample correlational survey. Journal of Clinical Nursing. 13 August 2019. PMid:31408560 https://doi.org/10.1111/jocn .15038

[4] Needleman J, Buerhaus P, Pankratz S, et al. Nurse staffing and inpatient hospital mortality.The New England Journal of Medicine. 2011; 364: 1037-45. PMid:21410372 https://doi.org/10.1056/NE JMsa1001025

[5] Spanager L, Ostegaard D, Lippert A, et al. Training of health-care employees in crisis resource management. 2013; 175(13): 880-4.

[6] World Health Organization. Patient safety curriculum guide. Multiprofessional edition. 2011.

[7] Salas E, Wilson KA, Burke CS. Using simulation-based training to improve patient safety: what does it take? Joint Commission Journal on Quality and Patient Safety. 2005 Jul; 31(7): 363-71. https://doi.org/10.1016/S1553-7250(05) 31049-X

[8] Ortega MC, Cecagno D, Llor AM, et al. Academic training of nursing professionals and its relevance to the workplace. Rev Lat Am Enfermagemv. 2015; 23(3): 404-410. PMid:26312632 https : //doi.org/10.1590/0104-1169.0432.2569

[9] Ostergaard H, Ostegaard D. Lippert A. Implementation of team training in medical education in Denmark. Quality and Safety. 2004.

[10] Hunter CL, Spence K, McKenna K, et al. Learning how we learn: an ethnographic study in neonatal intensive care unit. Journal of Advanced Nursing. 2008 Jun; 62(6): 657-64. PMid:18503649 https://doi.org/10.1111/j.1365-2648.2008.04632.x

[11] Coster S, Watkins M, Norman IJ. What is the impact of professional nursing on patients' outcomes globally? An overview of research evi- dence. International Journal of Nursing Studies. 2018 Feb; 78: 76-83. PMid:29110907 https://doi.org/10.1016/j.ijnurstu. 201 7.10 .009

[12] Kanyesiga JA, Bazinzi N. The impact of training and development on Job performance in Uganda Banking Sector. The Journal on Innovation and Sustainability. 2015.

[13] Aggarwal R, Mytton O, Derbrew M, et al. Training and simulation for patient safety. British Medical Journals Quality and Safety. 2010; 19(Suppl 2). PMid:20693215 https://doi.org/10.1136/qshc .2009 .038562

[14] Nassazi A. Effects of training on employee performance. Business Economics and Tourism [Online]. 2013.

[15] Alahmadi H. Assessment of patient safety culture in Saudi Arabian hospitals. Quality and Safety in Health Care. 2010; 19(5): e17. PMid:20430929 https://doi.org/10.1136/qshc.2009.0332 58

[16] Alkorashi A. Factors shaping patient safety, management in Middle East hospitals from nursing perspective: A Focus group study. Middle East Journal of Scientific Research. 2013; 15(10): 1375-1384.

[17] Harrison R, Cohen A, Walton M. Patient safety and quality of care in developing countries in Southeast Asia: A systematic literature review. International Journal for Quality in Health Care. 2015; 27(4): 240-254. PMid:26071280 https://doi.org/10.1093/intqhc /mzv041

[18] Al Mutair A. Quality of nursing care in Saudi Arabia. Journal of Nursing and Care. 2015; 4: 6. https://doi .org/10.4172/2167 $-1168.1000309$

[19] Truitt D. The effect of training and development on employee attitude as it relates to training and work efficiency. Sage Open. January 2011.

[20] Samra HA, McGrath JM, Rollins W. Patient Safety in the NICU: A Comprehensive Review. The Journal of Perinatal \& Neonatal Nursing. 2011; 25(2): 123-132. PMid:21540686 https ://doi .org/10 .1097/JPN . 0b013e31821693b2

[21] Ilias C, George M, Fotis V. Focusing on patient safety in the Neonatal Intensive Care Unit environment. Journal of Pediatric and Neonatal Individualized Medicine. 2017; 6(1): e060132. 